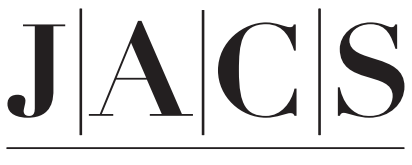

A R T I C L E S

Published on Web 07/08/2008

\title{
Rapidly Infrared-Assisted Cooperatively Self-Assembled Highly Ordered Multiscale Porous Materials
}

\author{
Zhongyu Zheng, ${ }^{\dagger}$ Kuiyi Gao, ${ }^{\dagger}$ Yanhong Luo, ${ }^{\dagger}$ Dongmei Li, ${ }^{\dagger}$ Qingbo Meng, ${ }^{\dagger, *}$ \\ Yuren Wang, ${ }^{\ddagger, *}$ and Daozhong Zhang ${ }^{\dagger}$ \\ Beijing National Laboratory for Condensed Matter Physics, Institute of Physics, Chinese \\ Academy of Sciences, 100190 Beijing, China, and National Microgravity Laboratory, Institute of \\ Mechanics, Chinese Academy of Sciences, 100080 Beijing, China
}

Received January 15, 2008; E-mail: qbmeng@ aphy.iphy.ac.cn; wangyr@imech.ac.cn

\begin{abstract}
In this paper, cooperative self-assembly (CSA) of colloidal spheres with different sizes was studied. It was found that a complicated jamming effect makes it difficult to achieve an optimal self-assembling condition for construction of a well-ordered stacking of colloidal spheres in a relatively short growth time by CSA. Through the use of a characteristic infrared (IR) technique to significantly accelerate local evaporation on the growing interface without changing the bulk growing environment, a concise three-parameter (temperature, pressure, and IR intensity) CSA method to effectively overcome the jamming effect has been developed. Mono- and multiscale inverse opals in a large range of lattice scales can be prepared within a growth time $(15-30 \mathrm{~min})$ that is remarkably shorter than the growth times of several hours for previous methods. Scanning electron microscopy images and transmittance spectra demonstrated the superior crystalline and optical qualities of the resulting materials. More importantly, the new method enables optimal conditions for CSA without limitations on sizes and materials of multiple colloids. This strategy not only makes a meaningful advance in the applicability and universality of colloidal crystals and ordered porous materials but also can be an inspiration to the self-assembly systems widely used in many other fields, such as nanotechnology and molecular bioengineering.
\end{abstract}

\section{Introduction}

The preparation of highly ordered porous materials with multiscale structures is of remarkable significance because of their unique and highly organized structures and properties, which have potential applications in a wide range of fields, from molecular separation, catalysis, membrane reactors, and biomaterials engineering to photovoltaics, photonic computing, and communications. ${ }^{1,2}$ Self-assembly (SA) provides a simple, costefficient, and powerful bottom-up approach for fabricating ordered arrays of building blocks at molecular, nanometer, and micrometer scales. An important example is the SA of colloids for preparing three-dimensional colloidal crystals (CCs) and ordered porous materials (OPMs), which has always attracted a great attention. ${ }^{2-7}$ In particular, cooperative self-assembly (CSA) of multiple colloidal components of different dimensions has a superior ability to produce intricate ordered structures at multiple length scales in one step. ${ }^{5,7}$ In another respect, CSA of multiscale colloids also serves as an excellent model system

\footnotetext{
$\dagger$ Institute of Physics, Chinese Academy of Sciences.

Institute of Mechanics, Chinese Academy of Sciences.

(1) (a) Yang, P.; Deng, T.; Zhao, D.; Feng, P.; Pine, D.; Chmelka, B. F.; Whitesides, G. M.; Stucky, G. D. Science 1998, 282, 2244-2246. (b) Yuan, Z.; Su, B. J. Mater. Chem. 2006, 16, 663-677.

(2) (a) Joannopoulos, J. D.; Villeneuve, P. R.; Fan, S. Nature 1997, 386, 143-149. (b) Nishimura, S.; Abrams, N.; Lewis, B. A.; Halaoui, L. I.; Mallouk, T. E.; Benkstein, K. D.; van de Lagemaat, J.; Frank, A. J. J. Am. Chem. Soc. 2003, 125, 6306-6310. (c) Lodahl, P.; van Driel, A. F.; Nikolaev, I. S.; Irman, A.; Overgaag, K.; Vanmaekelbergh, D. L.; Vos, W. L. Nature 2004, 430, 654-657.
}

for understanding the mechanism of self-organization phenomena, which occur widely in nature.

In the past decade, extensive efforts have been devoted to the SA of mono-CCs with better qualities and universality. ${ }^{6}$ In our previous work, SA of monosized colloidal spheres in a large

(3) (a) Jiang, P.; Hwang, K. S.; Mittleman, D. M.; Bertone, J. F.; Colvin, V. L. J. Am. Chem. Soc. 1999, 121, 11630-11637. (b) Vlasov, Y. A.; Bo, X. Z.; Strum, J. C.; Norris, D. J. Nature 2001, 414, 289-293. (c) Kubo, S.; Gu, Z. Z.; Takahashi, K.; Fujishima, A.; Segawa, H.; Sato, O. J. Am. Chem. Soc. 2004, 126, 8314-8319.

(4) (a) Holland, B. T.; Blanford, C. F.; Stein, A. Science 1998, 281, 538540. (b) Wijnhoven, J. E. G. J.; Vos, W. L. Science 1998, 281, 802804. (c) Juárez, B. H.; García, P. D.; Golmayo, D.; Blanco, A.; López, C. Adv. Mater. 2005, 17, 2761-2765. (d) Shimmin, R. G.; Vajtai, R.; Siegel, R. W.; Braun, P. V. Chem. Mater. 2007, 19, 2102-2107.

(5) (a) Meng, Q. B.; Fu, C. H.; Einaga, Y.; Gu, Z. Z.; Fujishima, A.; Sato, A. Chem. Mater. 2002, 14, 83-88. (b) Meng, Q. B.; Gu, Z. Z.; Sato, O.; Fujishima, A. Appl. Phys. Lett. 2000, 77, 4313-4315. (c) Wang, J.; Li, Q.; Knoll, W.; Jonas, U. J. Am. Chem. Soc. 2006, 128, $15606-15607$.

(6) (a) Zheng, Z. Y.; Liu, X. Z.; Luo, Y. H.; Cheng, B. Y.; Zhang, D. Z.; Meng, Q. B.; Wang, Y. R. Appl. Phys. Lett. 2007, 90, 051910. (b) Wong, S.; Kitaev, V.; Ozin, G. A. J. Am. Chem. Soc. 2003, 125 , 15589-15598. (c) Ye, Y. H.; LeBlanc, F.; Hache, A.; Truong, V. V. Appl. Phys. Lett. 2001, 78, 52-54. (d) Joannopoulos, J. D. Nature 2001, 414, 257-258. (e) Jiang, P.; Bertone, J. F.; Hwang, K. S.; Colvin, V. L. Chem. Mater. 1999, 11, 2132-2140.

(7) (a) Bartlett, P.; Ottewill, R. H.; Pusey, P. N. Phys. Rev. Lett. 1992, 68, 3801-3804. (b) Leunissen, M. E.; Christova, C. G.; Hynninen, A.-P.; Royall, C. P.; Campell, A. I.; Imhof, A.; Dijkstra, M.; van Roij, R.; van Blaaderen, A. Nature 2005, 437, 235-240. (c) Kitaev, V.; Ozin, G. A. Adv. Mater. 2003, 15, 75-78. (d) Wang, D.; Moehwald, H. Adv. Mater. 2004, 16, 244-247. (e) Bartlett, P.; Campell, A. I. Phys. Rev. Lett. 2005, 95, 128302. (f) Velikov, K. P.; Christova, C. G.; Dullens, R. P. A.; van Blaaderen, A. Science 2002, 296, 106-109. 
size range was investigated experimentally and theoretically. We developed the pressure-controlled isothermal heating vertical deposition (PCIHVD) method, ${ }^{6 a}$ a rapid, two-parameter (temperature and pressure) approach by which optimal growth conditions for SA of mono-CCs can be precisely obtained without limitations from materials and the dimensions of the colloidal spheres. Recently, excellent work on SA of binary CCs has also been reported by other groups. ${ }^{7}$ However, CSA of multiple colloidal particles has encountered three limitations: the sizes and materials of multiple colloids, the crystalline qualities of the resulting samples, and the long growth time. A crucial problem is that it is hard to achieve optimal growth conditions for CSA of multiple colloids with different dimensions; for example, CSA of nanoparticles and large colloidal spheres larger than $600 \mathrm{~nm}$ is still a challenge. ${ }^{5}$ In addition, the growth time is too long, usually lasting for several days or even several months. These three problems limit the diversity and practical applications of CCs and OPMs to a great extent. Breaking through scale and material limitations of the building blocks, improving the crystalline qualities, and reducing the growth time for CSA of multiple colloids simultaneously is an intellectual challenge; however, it is of urgent importance for the practical application of OPMs. Therefore, it is necessary to find a more efficient and universal method for achieving optimal growth conditions for fast CSA of multiscale colloids of all sizes and materials.

In our recent systematic studies of CSA of multiscale colloidal particles with the PCIHVD method, ${ }^{6 \mathrm{a}}$ it was found that a more complicated problem, the jamming effect, became evident in this CSA system at a relatively large growth rate. Jamming is a phenomenon in granular flow in which particles become arched and jammed at the opening before flowing through it when the ratio of the sizes of the opening and the particles reaches a critical value. ${ }^{8}$ Analogously, in the CSA system of multiscale colloids, this effect results from the space limitation imposed by the network of large colloidal spheres on the infiltration and transport of the small spheres in the vicinity of growing front. The jamming effect makes it difficult or even impossible to obtain the optimal growth conditions for the relatively fast CSA of multiscale colloids using the currently available methods, which require a dynamic balance among solvent evaporation, colloid transportation, and crystallization, as discussed later.

In this work, through the use of a characteristic infrared (CIR) technique, a fast, concise three-parameter (temperature, pressure, and IR intensity) approach called IR-assisted CSA (IACSA), which is based on the PCIHVD method, has been developed. This method involves irradiating the surface of the growing meniscus region with a CIR light while the growing temperature, vapor pressure, and CIR intensity are adjusted simultaneously. The CIR light used here has an irradiation-spectrum peak coinciding with the symmetric and asymmetric $\mathrm{O}-\mathrm{H}$ stretching vibration bands of liquid water $\left(3200-3450 \mathrm{~cm}^{-1}\right),{ }^{9}$ which can be intensively absorbed by water within a shallow penetration depth. ${ }^{10}$ Therefore, even at a low heating temperature in the

(8) (a) Corwin, E. I.; Jaeger, H. M.; Nagel, S. R. Nature 2005, 435, 1075 1078. (b) To, K.; Lai, P. Y.; Pak, H. K. Phys. Rev. Lett. 2001, 86, 71-74.

(9) Mayo, D. W.; Miller, F. A.; Hannah, R. W. Course Notes on the Interpretation of Infrared and Raman Spectra; Wiley-Interscience: New York, 2004; Chapter 11, pp 300-301.

(10) Mel'nikova, O. N.; Leont'yev, D. I.; Seliverstov, S. V.; Sluyev, M. V.; Petrov, V. P.; Shelkovnikov, N. K. Izv., Acad. Sci., USSR, Atmos. Oceanic Phys. (Engl. Transl.) 1996, 31, 564-567. whole growth cell, the local evaporation rate at the growing interface can be significantly increased to a value far beyond that under near-boiling conditions without interfering with the bulk growing environment. As a result, the jamming effect in the CSA can be effectively overcome. In addition, the whole growth system can readily reach the optimal conditions for SA of a well-ordered stacking of colloidal spheres by fast CSA of multiple colloids having largely different length scales. Moreover, the growth time can be remarkably shortened, from several hours for previous methods ${ }^{6 a, b}$ to $15-30 \mathrm{~min}$. Therefore, the three problems in CSA can be resolved simultaneously.

\section{Experimental Section}

In the CSA of composite CCs using the IACSA method, polystyrene (PS) colloidal spheres and silica nanoparticles were mixed together in aqueous solutions with different concentration ratios in glass growth vials. Glass microscope slides were cleaved into two equal pieces lengthwise, cleaned with anhydrous ethanol $(>99.7 \%)$ several times, and dried in a stream of nitrogen. After the full ultrasonic dispersion of the colloidal mixture of PS spheres and silica nanoparticles, the vial containing the mixed dispersion was immersed in an isothermal bath, and the clean glass substrate was fixed vertically in the center of the vial. Next, the pressurecontrolling system was started, and the surface of the growing meniscus was irradiated with characteristic IR light from an IR lamp. The temperature and pressure in the deposition vial became steady in $3 \mathrm{~min}$. Within 15-30 min, high-quality composite colloidal crystals were prepared. After removal of the PS template by calcination, ${ }^{5 \mathrm{a}, \mathrm{b}}$ silica OPMs were obtained from the original composite CCs. With the IACSA method, bimodal colloidal crystals (BCCs) were fabricated using mixed suspensions of $7 \mathrm{~nm}$ silica nanoparticles and PS microspheres with diameters ranging from $200 \mathrm{~nm}$ to $1.5 \mu \mathrm{m}$. Trimodal colloidal crystals (TCCs) with two different surface patterns were fabricated from mixed suspensions of silica nanoparticles and $180 \mathrm{~nm}$ and $1 \mu \mathrm{m}$ PS microspheres under different concentration ratios of the multiple colloids. The growth parameters under the optimal conditions for preparing BCCs and TCCs with this method are included in Table S1 and the surrounding text in the Supporting Information. After calcinations, highly ordered monoinverse opals (MIOs) and binary inverse opals (BIOs) were formed from the original bimodal and trimodal composite CCs, accompanied by a minor lattice structural shrinkage of $<5 \%$ due to dehydration of the nanoparticles. Monodispersed PS colloidal spheres with diameters ranging from $200 \mathrm{~nm}$ to $1 \mu \mathrm{m}$ were purchased from Duke Scientific Corporation, PS spheres with diameters of 180 and $1350 \mathrm{~nm}$ were provided by the groups of Prof. L. Jiang and Prof. Y. Song at the Institute of Chemistry, Chinese Academy of Sciences, and silica nanoparticles with a mean size of $7 \mathrm{~nm}$ were purchased from Catalysts and Chemicals Industries Co., Ltd.

\section{Results}

Figure 1 presents SEM images of the (111) surfaces of MIOs with different pore sizes. They were prepared from silica nanoparticles and PS spheres with diameters of 451 and 596 $\mathrm{nm}$ and 1 and $1.35 \mu \mathrm{m}$ using the IACSA method. In these samples, perfect hexagonal close packing of air spheres can be observed, and the absence of as-grown defects in the largely observed area is apparent. Beneath each air sphere in the first layer, a regular triangular pattern of black pores connecting the sphere with the three below it are shown, revealing the highly ordered face-centered cubic (fcc) structure of the MIOs, which have single-crystal domains several millimeters in size with few point defects and line dislocations. The SEM images give an average of $\sim 10^{-3}$ dislocations and $\sim 10^{-4}$ point defects per unit cell [i.e., one dislocation and point defect for every $\sim 10^{4} \mu \mathrm{m}^{2}$ 


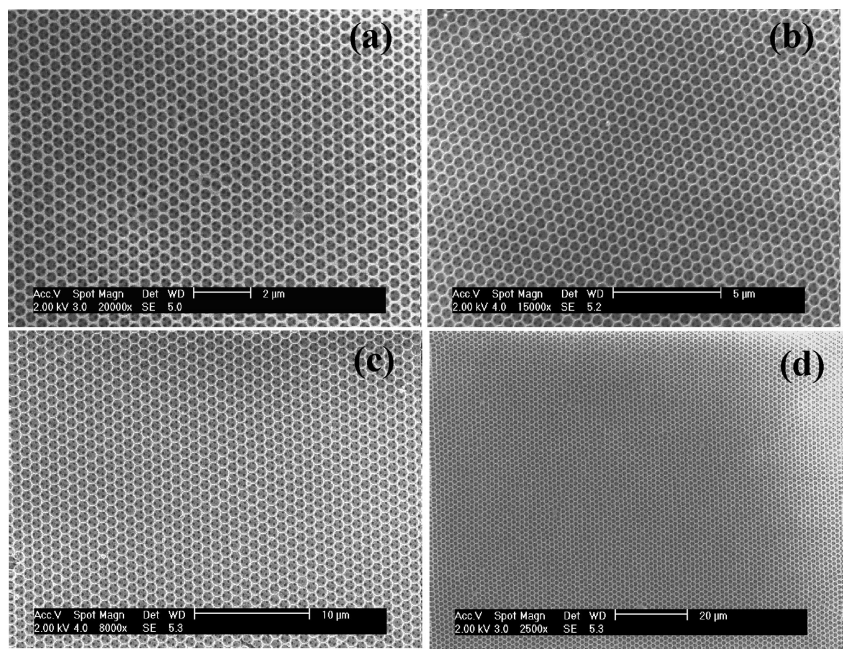

Figure 1. Top-view SEM images of silica MIOs prepared from silica nanoparticles and PS spheres with diameters of (a) $451 \mathrm{~nm}$, (b) $596 \mathrm{~nm}$, (c) $1 \mu \mathrm{m}$, and (d) $1.35 \mu \mathrm{m}$ using the IACSA method.
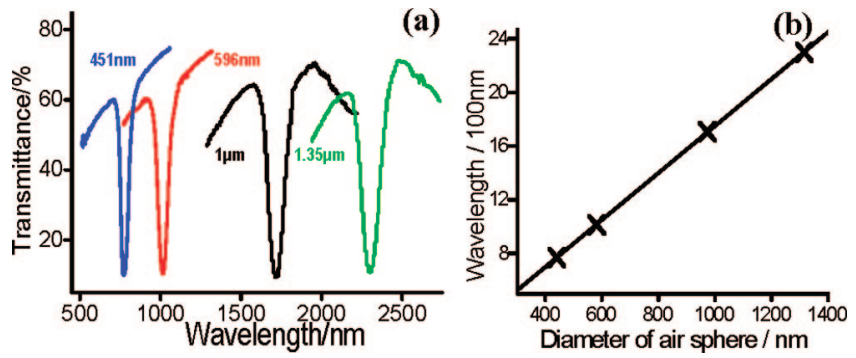

Figure 2. (a) Transmittance spectra for the silica MIOs shown in Figure 1. (b) Linear relation between the PBG positions and air-sphere sizes of the MIOs.

of area on the (111) surface]. The dislocations usually extend no more than tens of unit cells (several micrometers). The order and orientation of the intact single crystals is not appreciably diminished by such small amounts of localized dislocations and point defects. These results indicate that the crystalline regularity of the samples is superior to that of MIOs fabricated by template-filling methods $\left(\sim 1 \%\right.$ stacking faults and $\sim 10^{-3}$ point defects per unit cell). ${ }^{3 \mathrm{~b}}$ The optical properties of the MIOs from Figure 1 were characterized by vis-near-IR transmittance spectra with the incident light normal to the fcc (111) plane (Figure 2a). For all of the samples, the depths of the photonic band gaps (PBGs) exceeded 60\%, which reveals that the high optical qualities do not decline with increasing size of the air spheres. To the best of our knowledge, such high optical qualities (deep PBGs) for MIOs, which benefit from the high degree of long-range ordering and the lack of defects in our MIOs, have never been reported by other groups. In addition, the positions of the PBGs in these spectra were 771, 1013, 1708, and $2300 \mathrm{~nm}$, respectively, which scaled well with the air-sphere sizes of the MIOs (Figure 2b). By means of Bragg's law, the positions of the PBGs for the MIOs could also be calculated from the diameters of the air spheres and the effective refractive index of the silica-air matrix, and the results were in accordance with the measured values (see the Supporting Information).

Figure 3a shows a low-magnification SEM image of the (111) surface of a silica MIO prepared from $1 \mu \mathrm{m}$ PS colloidal spheres using the IACSA method. The parallel lines are known as Moiré fringes and are induced by interactions between a scanning line pattern of an electron microscope and hexagonal lattices of

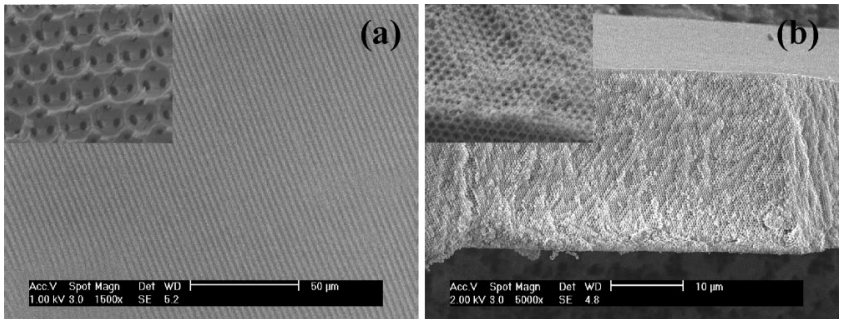

Figure 3. (a) Low-magnification top-view SEM image and (inset) a fracture image of a silica MIO prepared from $1 \mu \mathrm{m}$ PS colloidal spheres. (b) SEM image and (inset) a magnified image of the cross section of a silica MIO prepared from $451 \mathrm{~nm}$ PS colloidal spheres.

colloidal spheres at a specific magnification. ${ }^{11}$ The well-defined, uniform, large-area linear Moiré pattern exhibits the highly ordered hexagonal surface packing in single domains (with dimensions of at least $0.2 \times 0.2 \mathrm{~mm}^{2}$ ) of these MIOs. Cracks formed during the drying process were significantly reduced in these samples, with intact films spreading over several millimeters. The magnified SEM fracture image in the inset of Figure 3a shows the detailed structure of an interior (111) layer in the MIO film, which further manifests the highly ordered fcc structure inside. In a single deposition, the thickness of the MIOs can altered from several to nearly 100 layers, which is several times greater than the maximal thickness of the opals obtained by other convective SA methods. ${ }^{3 a, b}$ Figure $3 b$ shows a cross-section SEM image of an MIO prepared from $451 \mathrm{~nm}$ PS colloidal spheres, in which the high degree of vertical ordering was well maintained throughout the whole thickness of nearly 80 layers in the film. The magnified image in the inset shows in detail the regular packing of air spheres in the cross section of the MIO. Such highly vertical ordering in intact single domains with significantly large areas is responsible for the deep PBGs of these MIOs. The perfect lattice structure and superior optical quality of the resulted samples have confirmed the success of this method.

Figure 4a,b shows large-area top-view SEM images of silica BIOs prepared with two different surface patterns. Figure $4 c, d$ presents high-magnification SEM images of the samples shown in Figure 4a,b, respectively, which demonstrate their detailed hierarchical porous structures. In the surface pattern shown in Figure 4c, the small air spheres form a regular triangular pattern in the interstitial voids (highlighted by the white circle), while the pattern shown in Figure $4 \mathrm{~d}$ is similar except for the additional single small sphere (two such spheres are highlighted by blue arrows) between each pair of neighboring large spheres and just above the pair of vertically opposite small-sphere triplets. These two surface patterns indeed represent the same hierarchical porous structure of the prepared BIOs. In these samples with fcc lattice structures, each quadruplet of large air spheres constitutes a tetrahedron, while each sextuplet constitutes an octahedron (denoted by the red and white lines, respectively, in Figure 4d). The three large air spheres denoted by the red triangle and the one just below them in the second layer form a quadruplet. The three large air spheres denoted by the solid white triangle and the three in the second layer denoted by the dashed white triangle form a sextuplet. The three-dimensional models in Figure $4 \mathrm{e}-\mathrm{h}$ simulate the filling of small spheres into the hollow tetrahedral and octahedral sites in the large-sphere network. Each hollow tetrahedral site of a large-sphere qua-

(11) Subramania, G.; Constant, K.; Biswas, R.; Sigalas, M. M.; Ho, K.-M. Adv. Mater. 2001, 13, 443-446. 

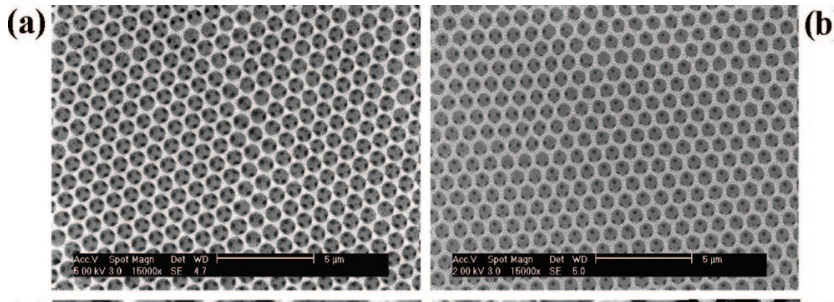

(c)
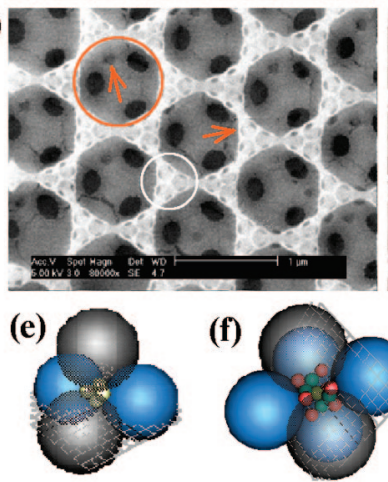

(g)
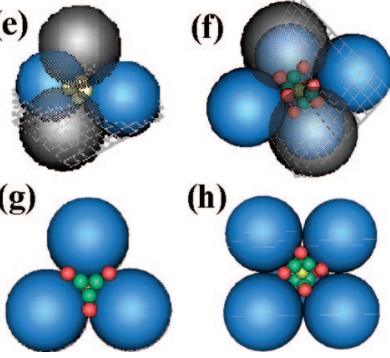

(h)

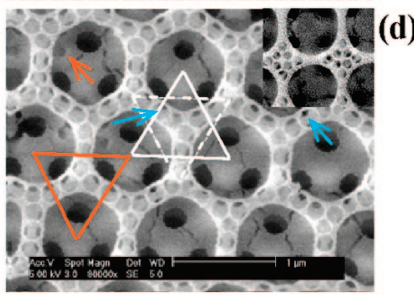

(i)

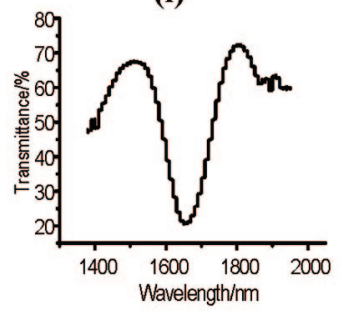

Figure 4. Large-area top-view SEM images of BIOs with different surface patterns prepared from $7 \mathrm{~nm}$ silica nanoparticles and (a) $200 \mathrm{~nm}$ and (b) 1 $\mu \mathrm{m}$ PS spheres using the IACSA method and (c, d) corresponding magnified SEM images, with the inset in (d) showing the (100) surface SEM image. Simulated models for filling of the (e) tetrahedral and (f) octahedral sites of the large-sphere network by small spheres. (g, h) Models for the (111) and (100) surfaces of the octahedral site shown in figure (d) and its inset, respectively. (i) Transmittance spectrum for these BIOs.

druplet contains a small-sphere quadruplet, and each hollow octahedral site can hold 15 small spheres: eight in the outer layer (red), six in the middle layer (green), and one in the center (yellow). The additional small spheres in the latter surface pattern highlighted by blue arrows in Figure 4d correspond to the red ones in the outer layer within the octahedral site in Figure $4 \mathrm{~g}$, which are also the ones outside the tetrahedral site. The inset in Figure 4d is an SEM image of the (100) surface of an octahedral site in the BIO, in which the small air spheres are packed the same as in the simulated model in Figure 4h. In these BIOs, each large air sphere (L) is interconnected with 12 neighboring ones by large macropores (exemplified by the three large black windows in the red circle), indicating that the large air spheres are organized in highly ordered fcc packing. The small air spheres (S) regularly occupy the interstitial voids between large spheres and connect with them by small macropores (such as the small black windows designated by red arrows). These small spheres are also interconnected with mesopores (highlighted by the white circle). These BIOs have a magnified lattice structure like the one reported by Wang et al., ${ }^{5 c}$ which has an average crystal stoichiometry of $\mathrm{LS}_{21-23}$, but our BIOs have been formed over a much larger range of length scales, from nanometer through submicrometer to micrometer. Moreover, from the large-area top-view SEM images of the BIOs with the two surface patterns (Figure 4a,b), it can be seen that the surface pattern stays consistent very well across the same sample. This reveals the superiority of our OPMs, due to the high stability and reproducibility of the optimal growth conditions in the IACSA process, which can clearly reflect the actual effect of any changes of each factor in

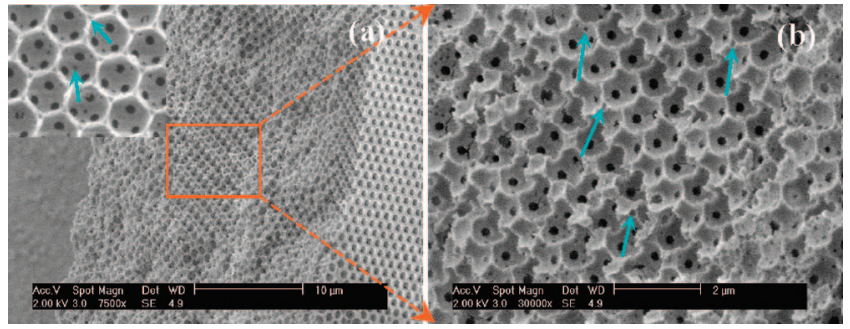

Figure 5. (a) Cross-section SEM image of the BIOs prepared from 200 $\mathrm{nm}$ and $1 \mu \mathrm{m}$ PS spheres and $7 \mathrm{~nm}$ silica nanoparticles using the IACSA method, with a fracture image in the inset. (b) Magnified cross-section SEM image of the rectangular area in (a).

growing environment (e.g., the concentration ratio of multiple colloids in the present case). The transmittance spectra for the prepared BIOs were also measured. It was found that the BIOs with two surface patterns have the same PBG position of 1664 $\mathrm{nm}$, as observed from the representative result shown in Figure 4i. Additionally, upon consideration of the close packing of the silica nanoparticles in the voids, their volume fraction in these BIO structures was calculated from the PBG position according to Bragg's law and found to be consistent with an average crystal stoichiometry of $\mathrm{LS}_{21-23}$. These results further confirm that these BIOs have the same hierarchical porous structures as analyzed above. Moreover, the depth of photonic band gap exceeded 50\%, which indicates that the high degree of ordering in these BIOs does not decline as a result of the insertion of small spheres into the interspaces between large spheres.

Figure 5a shows an SEM image of the cross section for the BIOs, in which highly ordered vertical packing can be seen through out the whole thickness. In the magnified cross-section image for the rectangular area (Figure 5b) and the fracture image (inset of Figure 5a), the small black holes highlighted by green arrows reveal the distribution of small air spheres throughout the samples. Consequently, with the IACSA method, BCCs and TCCs with two regular surface patterns have been prepared from mixed colloids of PS microspheres with diameters of $200 \mathrm{~nm}$ to $1.5 \mu \mathrm{m}$ and silica nanoparticles. In a single deposition, the film thickness can be varied from several to nearly 100 layers by changing the total concentration of the colloidal mixture. After removal of the PS spheres by calcination, superior-quality large-area MIOs and hierarchical porous structures at length scales varying over an extremely large range (from nanometer through submicrometer to micrometer) can be formed from the original composite CCs.

\section{Discussion}

To provide a comprehensive understanding of the process of IACSA, the characteristics of colloids and the mechanism of SA of CCs should be discussed first. Figure 6a presents a schematic illustration of the SA of colloids with vertical deposition. The solution wets the substrate, forming a meniscus region. Solvent evaporation from the meniscus induces capillary flow, called "Marangoni flow", ${ }^{12}$ which must have sufficient power to drive the transport of colloids from the supporting suspension to the meniscus, and the interparticle capillary forces which cause SA of the colloids into close-packed arrays. Consequently, three processes coexist in the SA system: solvent evaporation, colloid transportation, and crystallization, with the latter two dependent on the solvent evaporation rate. As confirmed in our PCIHVD experiments and

(12) Myers, D. Surfaces, Interfaces, and Colloids: Principles and Applications; Wiley-Interscience: New York, 1999; Chapter 6, pp $101-118$. 


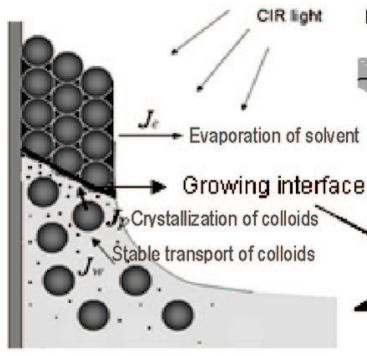

(a)

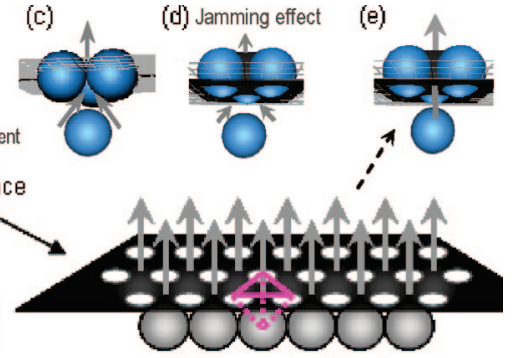

(b) Enhanced capillary flow
Figure 6. Schematic illustrations of (a) the growing process and (b) the trapping of colloidal spheres in the optimal positions on the growing interface induced by the enhanced capillary flows in IACSA of colloidal spheres and nanoparticles. The colloidal sphere quadruplet highlighted by the red lines in (b) is shown in (e). (c) Straightway capillary flow at the growing interface in SA of monosized colloidal spheres and (d) the jamming effect in CSA of colloidal spheres and nanoparticles using the PCIHVD method. The lengths of the gray arrows in $(b-e)$ scale with the flux and velocity of capillary flow.

the work of others, ${ }^{6}$ in order to grow CCs with high crystalline quality, the three processes should keep a dynamical balance, which requires two crucial conditions: an optimal evaporation rate and a uniform dispersion of the colloidal particles in suspension. For SA of monosized colloidal spheres over a large size range (100nm-1 $\mu \mathrm{m})$, it is easy to obtain common optimal conditions because the interstitial channels between spheres are scaled with the sphere diameters, which are large enough to ensure straightway capillary flow. Colloids are driven by the capillary flow to the optimal positions for forming a close-packed lattice structure, as shown in the schematic of a mono-CC growing interface in Figure 6c. However, in the case of CSA of microcolloidal spheres and nanoparticles, there is an additional problem. As the schematic of the growing interface in this CSA system (Figure 6d) indicates, the channels between colloidal spheres are jammed by the interstitial nanoparticles, causing the liquid flow to swirl before entering the meniscus and thereby significantly reducing the capillary flux. Thus, the colloids do not have enough energy to find the optimal positions and become locked into a disordered configuration; in addition, even the transport of colloids can be impeded. Therefore, the dynamic balance mentioned above is destroyed, making it difficult to obtain the optimal growth conditions for CSA of multiple CCs. This jamming effect exists in CSA systems involving multiscale colloids and gradually becomes more serious as the ratio of the sizes of the various colloidal particles increases. In particular, the jamming effect acts evidently in the CSA of multiscale colloids at a relatively fast growth rate, such as in PCIHVD, which has been shown to be inapplicable to CSA of microcolloidal spheres and nanoparticles. For small PS spheres, the quality of the resulting MIOs is extraordinarily poor, and for PS spheres larger than $600 \mathrm{~nm}$, the CSA cannot even be accomplished (detailed information concerning this is presented in the Supporting Information).

In this regard, the IACSA method provides great benefits. First, by application of the CIR irradiation, the local evaporation rate in the growing interface can be controllably increased far beyond that under near-boiling conditions without changing the bulk growing environment. Thus, enhanced capillary flow between colloidal spheres on the growing front can be induced, which can break the arched structure of the nanoparticles and draft them through the interstitial voids. Also, colloidal spheres in the vicinity of the growing front can be steered to the optimal positions, as illustrated in Figure 6b,e. Consequently, the jamming effect can be effectively overcome, restoring the dynamical balance in the CSA system.
Moreover, by adjusting the parameters in a three-dimensional phase space defined by temperature, vapor pressure, and CIR intensity, the evaporation rate can be changed greatly, allowing the optimal growth conditions for CSA of multiple colloids with any sizes to be concisely obtained. However, this is difficult or even impossible in previous one- or two-parameter SA methods, wherein the growth conditions are limited along a line or even to a point. ${ }^{6}$ Second, the growth time can be remarkably reduced to $15-30 \mathrm{~min}$, which makes this method more favorable for industrial applications. Third, the CIR light also serves as a heating source, which can cause a positive temperature gradient from the supporting water to the meniscus. As a result, a steady, fast liquid flow, i.e., a steady transport of colloidal spheres to the meniscus, can be formed in the vicinity of the growing interface,,$^{13}$ which can ensure the high stability and reproducibility of the CSA process. Finally, this approach inherits all the advantages of all of the previous methods on which it is based. For example, if the strong near-boiling convective flow in dispersions applied in IHEISA method ${ }^{6 \mathrm{~b}}$ is maintained, the gravity sedimentation of large colloidal spheres can be successfully counteracted. Moreover, just as in the PCIHVD method, ${ }^{6 a}$ adjusting the growth temperature as necessary to avoid distortion and damage of the material properties of colloids can greatly extend the range of suitable building-block materials to include, for example, organic and bioactive materials. Consequently, the three limitations in CSA of multiscale colloids can be successfully overcome at the same time with this simple strategy.

\section{Conclusion}

We have developed a concise method called IR-assisted CSA (IACSA), whereby the application of CIR light in order to significantly accelerate local solvent evaporation on the growing interface and form a steady, fast liquid flow to the meniscus has allowed the three challenging problems in CSA of multiscale colloids to be successfully overcome simultaneously. Superior quality mono- and hierarchical inverse opals over a large range of length scales (from nanometer to micrometer) have been prepared within a remarkably reduced growth time using this method. More importantly, this method enables optimal CSA conditions to be achieved without limitations on materials and sizes of colloidal particles. We believe that because of their superior quality and diversity, CIR-assisted self-assembled CCs and OPMs represent meaningful advances in applicability and universality. The IACSA technique also can be an inspiration to many other SA systems widely used in the fields of nanotechnology and molecular bioengineering.

Acknowledgment. This work was supported by the National Natural Science Foundation of China (Grants 20725311, 20673141, 20703063, and 20721140647), the Ministry of Science and Technology of China (973 Project, Grant 2006CB202606, and 863 Project, Grant 006AA03Z341), and the 100 Talent Project of the Chinese Academy of Sciences.

Supporting Information Available: Detailed information on the SEM images of MIOs made by the PCIHVD method, schematic setup of the IACSA method, optimal growth parameters for CSA of BCCs and TCCs using the IACSA method in our experiments, and results of theoretical calculations on the positions of PBGs for the prepared MIO and BIO structures. This material is available free of charge via the Internet at http:// pubs.acs.org.

\section{JA800327N}

(13) Tritton, D. J. Physical Fluid Dynamics; Van Nostrand Reinhold Company: New York, 1977. 